\title{
Management of acute and chronic aortic disease during the COVID-19 pandemic- results from a web-based ad-hoc platform
}

\author{
Martin Czerny ${ }^{1}$, Josua VanDenBerg ${ }^{2}$, Roberto CHIESA ${ }^{3}$, Michael Jacobs ${ }^{4}$, Stephan Jakob ${ }^{5}$, \\ Hans-Joerg Jenni ${ }^{6}$, Roberto Lorusso ${ }^{7}$, Davide Pacini ${ }^{8}$, Eduard Quintana ${ }^{9}$, B. Rylski ${ }^{1}$, Klaus \\ Staier $^{1}$, Nikolaos Tsilimparis ${ }^{10}$, Thomas Wyss $^{11}$, Roman Gottardi ${ }^{12}$, and Juerg Schmidli ${ }^{13}$ \\ ${ }^{1}$ University Heart Center Freiburg- Bad Krozingen, Germany \\ ${ }^{2}$ Ospedale Regionale di Lugano, Lugano, Switzerland \\ ${ }^{3}$ Vita-Salute San Raffaele University, San Raffaele Scientific Institute \\ ${ }^{4}$ University Hospital Maastricht, The Netherlands \\ ${ }^{5}$ Bern University Hospital, University of Bern, Switzerland \\ ${ }^{6}$ Inselspital, Bern University Hospital, University of Bern, Switzerland \\ ${ }^{7}$ Maastricht University Medical Centre \\ ${ }^{8}$ Policlinico S.Orsola-Malpighi, Bologna \\ ${ }^{9}$ Hospital Clínic \\ ${ }^{10}$ Ludwig-Maximilians-University Munich \\ ${ }^{11}$ Hospital Winterthur, Switzerland \\ ${ }^{12}$ Mediclin Heart Centre,Lahr \\ ${ }^{13}$ University Bern
}

September 1, 2020

\begin{abstract}
Background. To share the results of a web-based expert panel discussion focusing on the management of acute and chronic aortic disease during the COVID-19 pandemic. Methods. A web-based expert panel discussion on April 18 th 2020 where 8 experts were invited to share their current experience with COVID-19 disease touching several aspects of aortic medicine, was performed. After each talk, specific questions were asked to the online audience and results were immediately evaluated and shared with faculty and participants. Results. As of April 18 2020, 73.3\% of the 87 participants from 26 different countries answered that overall less than 200 COVID positive patients have been treated in their respective institutions. Sixty-five percent reported that their hospital was well prepared for the pandemic. In $57.7 \%$, the percentage of infected health care professionals was below $5 \%$ whereas $23 \%$ reported $5-10 \%$ and $19.2 \%$ reported between 10 and $20 \%$. Three percent reported to have seen aortic ruptures in primarily elective patients having been postponed because of the anticipated need to provide sufficient ICU capacity because of the pandemic. Nearly $70 \%$ reported a decrease of acute aortic syndromes since the start of the pandemic. Conclusions. The current COVID-19 pandemic has- led to a decrease of referrals of acute aortic syndromes in many services. The reluctance of patients seeking medical advice seems to be a major driver. The number of patients who have been postponed due to provisioning ICU resources but having experienced aortic rupture in the waiting period, is still low.
\end{abstract}

\section{Hosted file}

Aorta and COVID-19 31.08. RG without tables.doc available at https://authorea.com/users/ 355300/articles/478611-management-of-acute-and-chronic-aortic-disease-during-the-covid19-pandemic-results-from-a-web-based-ad-hoc-platform 
1 Figure 1. Referrals within the first 6 weeks

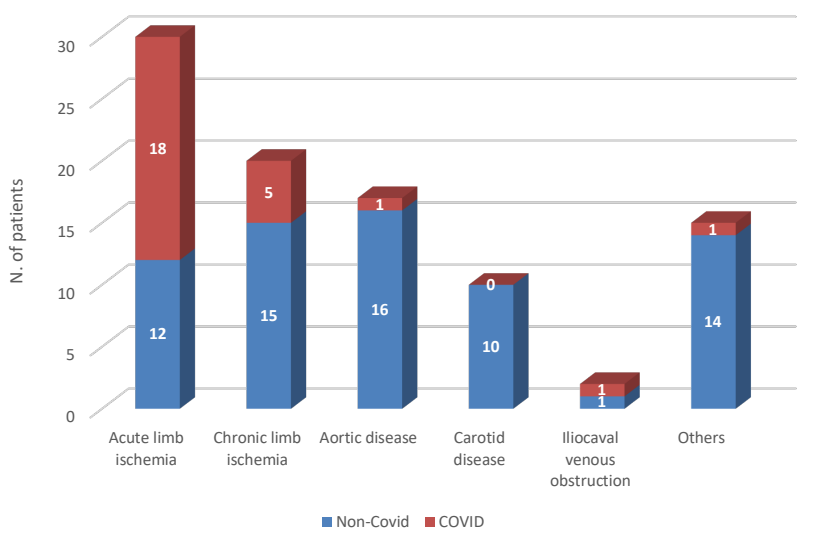

3 
1 Figure 2. CTA after TEVAR in a COVID-19 patient showing typical ground class opacities

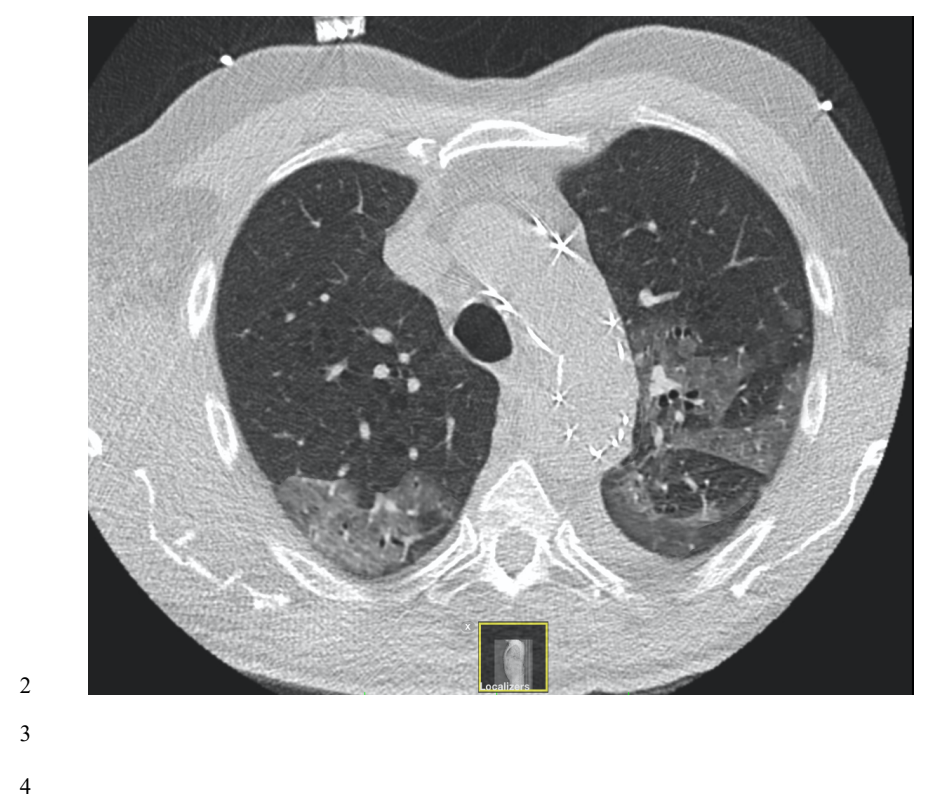


1

2 Figure 3. CTA of a patient with acute type A aortic dissection and suspicion of COVID-19

3 disease due to unspecific pulmonary infiltrates

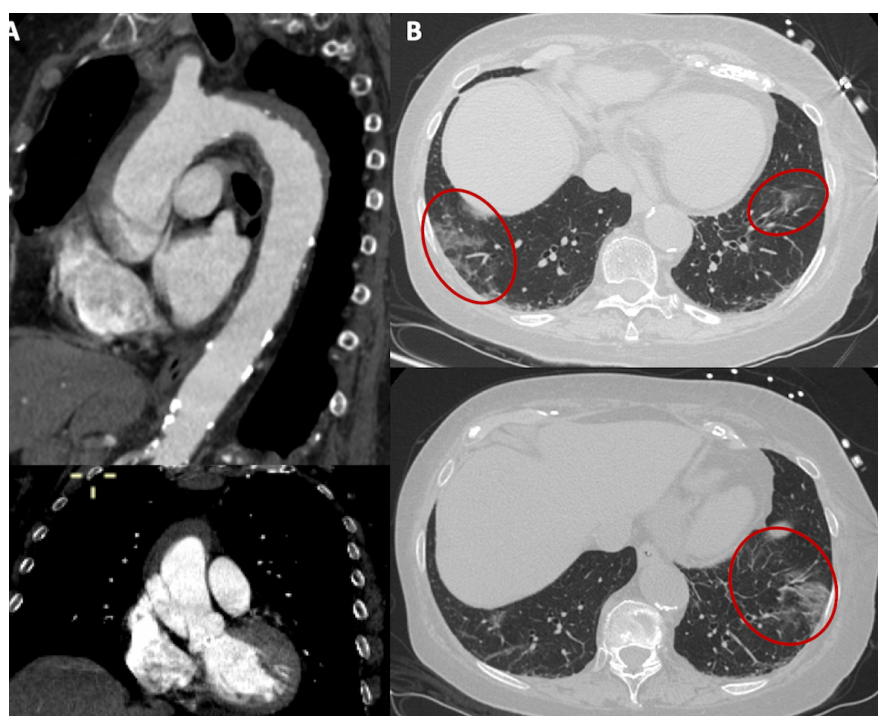


2 Figure 4. CTA after total endovascular aortic arch repair for acute type A aortic dissection

3

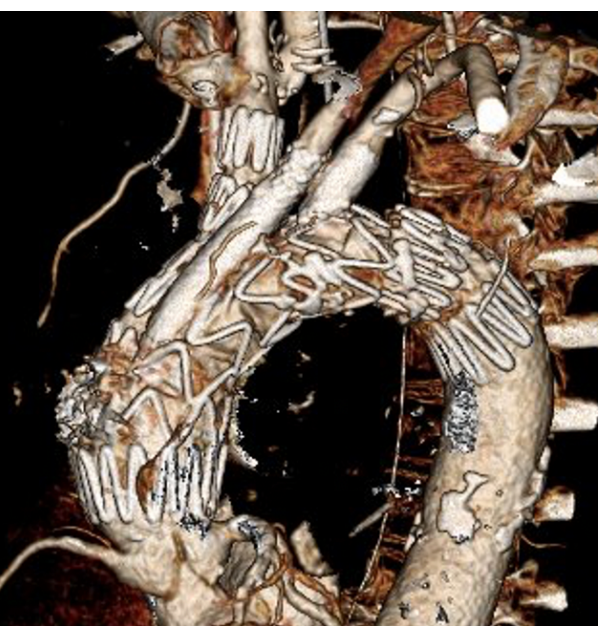


1

2 Figure 5. Questions and answers 1-4

3
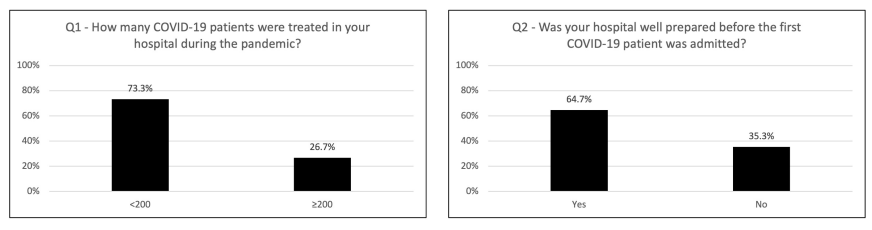

4
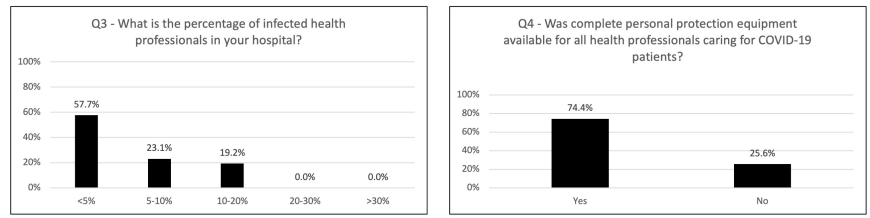
1

2 Figure 6. Questions and answers 5-8
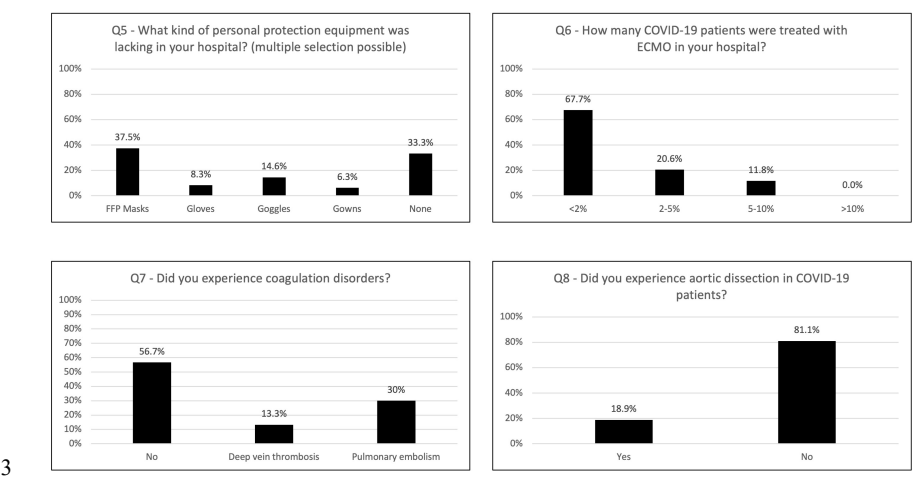

4 
1

2 Figure 7. Questions and answers 9-12
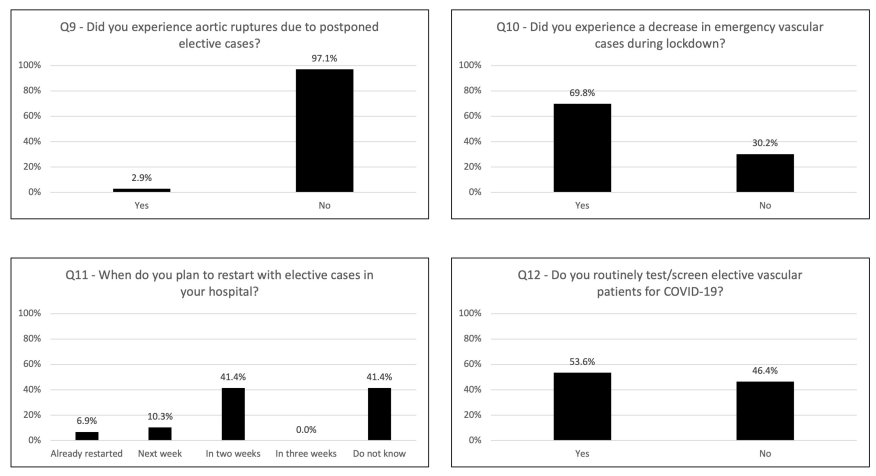

4 